\title{
Nonlinear plasmonics of metallic heptamers
}

\author{
Krishnan Thyagarajan, Simon Rivier and Olivier J.F. Martin \\ Nanophotonics and Metrology Laboratory (NAM), \\ Swiss Federal Institute of Technology Lausanne (EPFL), 1015 Lausanne, Switzerland
}

\begin{abstract}
Second-harmonic generation (SHG) from centrosymmetric nanostructures originating from the breaking of inversion symmetry at their surfaces is a well-known phenomenon and is extensively used as a surface probe in nonlinear optical microscopy. In recent years, SHG and its subsequent enhancement using plasmonics has been observed from nanostructures such as sharp metallic tips, nanoantennae and nanodimers. However, the process is still inefficient, its mechanism not well understood, and an improvement is required. In order to achieve a higher conversion efficiency, we investigate experimentally a way to minimize the radiative losses at the fundamental frequency. In the present investigation, we use silver heptamer nanostructures and tune the subradiant mode of the Fano resonance to the fundamental of the pump source, while tuning a higher order multipolar term to the second harmonic and in the process we obtain a significant enhancement of the second harmonic signal. A detailed explanation and analysis of this is provided by considering the contribution and effect of varying different parameters, such as gap size and radius, as well as the overall symmetry of the structure. In fact, recently gold heptamers have been studied and have indeed shown strong hybridization of their constituent resonant primitive plasmonic modes, leading to new hybridized superradiant 'bright' and subradiant 'dark' modes ${ }^{1,2}$. The ease of fabrication and possible tunability achievable, make these structures very versatile tools for studying surface SHG in nanostructures.
\end{abstract}

Keywords: Nonlinear plasmonics, second harmonic generation (SHG), plasmonic oligomers, Fano resonance

\section{INTRODUCTION}

Second-harmonic generation (SHG) from centrosymmetric nanostructures originating from the breaking of inversion symmetry at their surfaces is a well-known phenomenon. Recently, complex nanostructures such as gold heptamers have been studied and have shown strong hybridization of their constituent resonant primitive plasmonic modes, leading to new hybridized superradiant 'bright' and subradiant 'dark' modes. The incident photons excite the bright mode, which couples to the dark mode. Interference between both modes leads to the Fano resonance in these systems ${ }^{3}$. One technique to possibly improve the SHG is to take advantage of these two qualitatively different modes constituting the Fano resonance.

In this work, we investigate silver heptamers and tune the subradiant mode of the Fano resonance to the fundamental of the pump source and a multipolar mode to the second harmonic, by changing various parameters including the overall symmetry of the structure, in order to achieve a more efficient generation of the second harmonic. Typically, the bright and dark modes in a Fano resonance are spectrally separated by $50-100 \mathrm{~nm}$. Therefore it is difficult to tune the same structure to have the subradiant dark mode at the fundamental, while having the superradiant bright mode at the second harmonic. The next best solution is to tune a radiant multipolar mode to the second harmonic, while having the fundamental at the dark mode, which is what we have attempted in this work. Although, in our simulations, we have not included a substrate, the inclusion of a dielectric substrate would only result in a red-shift in the individual plasmon resonances, due to the screening charges that are induced at the substrate-metal interface ${ }^{4}$.

Photonic and Phononic Properties of Engineered Nanostructures II,

edited by Ali Adibi, Shawn-Yu Lin, Axel Scherer, Proc. of SPIE Vol. 8269

82691Z - (C) 2012 SPIE · CCC code: 0277-786X/12/\$18 - doi: 10.1117/12.907352

Proc. of SPIE Vol. $826982691 Z-1$ 


\section{PLASMON HYBRIDIZATION IN PLASMONIC OLIGOMERS}

Metallic heptamers are versatile nanostructures that can be used to effectively study SHG, taking advantage of not only the symmetry-breaking possible in these structures, but also the Fano resonances due to the strong hybridization ${ }^{5-7}$. The heptamer can be visualized as a hexamer ring with a monomer at the center. The primary plasmonic modes that are excited in these two structures - the hexamer and the monomer - are dipolar in nature, although slightly displaced with respect to each other, due to the onset of interaction among the monomers that constitute the hexamer. When the monomer is placed in the center of the hexamer ring, there is a drastic impact on the scattering cross-section of the resulting heptamer, which now exhibits the well-known Fano dip. This can be explained using the plasmon hybridization model $^{8,9}$ - due to the close proximity of the hexamer and the monomer at the center, the primitive dipolar plasmonic mode of the hexamer ring hybridizes with the primitive dipolar plasmonic mode of the central monomer, producing a collective superradiant 'bright' mode and a collective subradiant 'dark' mode as seen in Fig. 1.

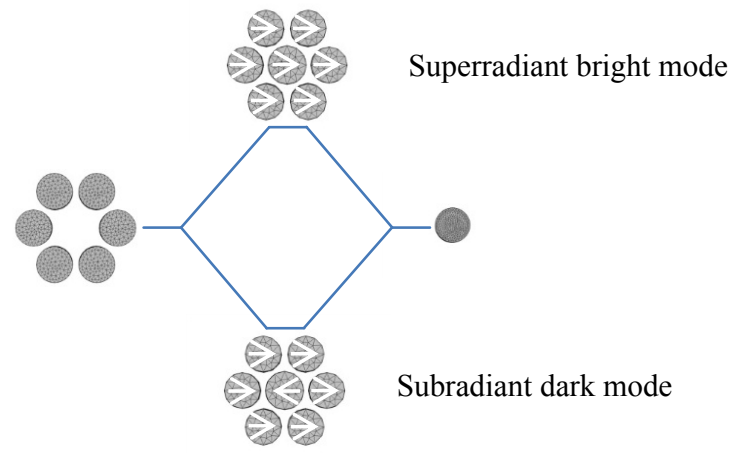

FIG. 1: A schematic of the plasmon hybridization picture of the hexamer and monomer forming the collective modes of the heptamer.

The two collective modes differ qualitatively in their ability to couple to the incoming field and to radiate back into the far-field. The superradiant mode is a mode in which the dipoles in all the seven monomers oscillate in phase, leading to a strong coupling to the incoming field and a strong radiation into the far-field. While the subradiant mode, is a mode in which the central monomer has its dipole oscillating out of phase with respect to the dipoles in the ring, causing a weak coupling to the incoming field and a weak radiation into the far-field. The asymmetric Fano resonance can be visualized as the destructive interference of a discrete state with a possible continuum of states and is observed in a range of natural phenomena. The interference occurs between two possible paths that excite the system from an initial to a final state. In the system considered in this work, the initial system consists of the unexcited superradiant mode and subradiant collective mode, while the final system involves an excited superradiant mode. The continuum of states is provided by the incident photons impinging on the system and directly excites the superradiant mode, while the subradiant mode can be excited only by the near-field coupling to the superradiant mode. The excited subradiant mode then re-excites the superradiant mode and it is the interference of these two pathways to excite the superradiant mode that causes the Fano dip. At this resonance, a mode with low radiative losses (the subradiant mode) is excited, which can be exploited to efficiently generate the second harmonic signal. The adjustment of the geometrical parameters allows for the excitation of a multipolar mode at the second harmonic, which can possibly couple to the dark mode, due to its spectral and spatial overlap. As is discussed in some earlier work, it is possible to tune the features of the Fano resonance by changing various geometrical parameters such as thickness, radius or gap dimensions, angle of incidence ${ }^{5-7}$. For our proposed experimental setup consisting of a Ti:Sapphire femtosecond laser, we are able to engineer the heptamers such that we can shift the radiant bright mode to $415 \mathrm{~nm}$ and the subradiant dark mode (and the Fano dip) to a fundamental pump wavelength of $830 \mathrm{~nm}$, as can be seen in Fig. 2. 


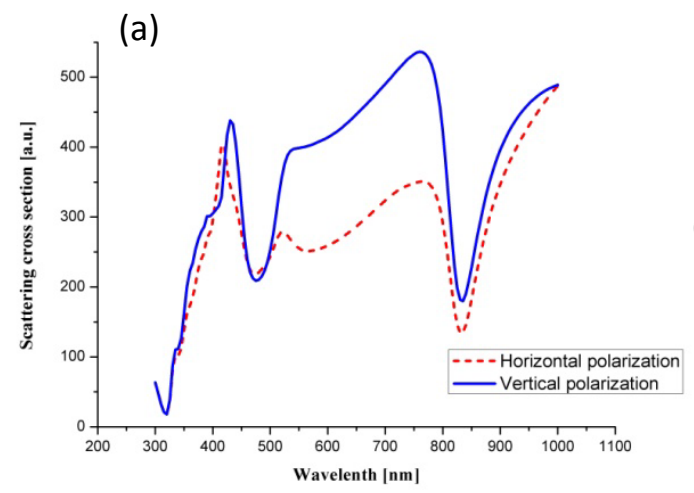

(b)

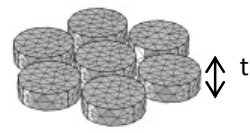

(c)

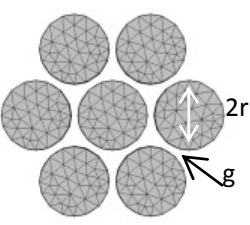

FIG. 2: (a)Scattering cross section of symmetric silver heptamer with radii (r) $100 \mathrm{~nm}$, thickness (t) $60 \mathrm{~nm}$ and a gap (g) of $20 \mathrm{~nm}$; (b) and (c) simulated structures

Looking at the possible dipole distribution within the individual monomers that constitute the heptamer when light is incident on the sample, we can see the participation of higher order modes in the formation of the radiant mode at the spectral position of $415 \mathrm{~nm}$ and the out of phase central dipole oscillation in the subradiant mode at the spectral position of $830 \mathrm{~nm}$ as can be seen in figure 3 .
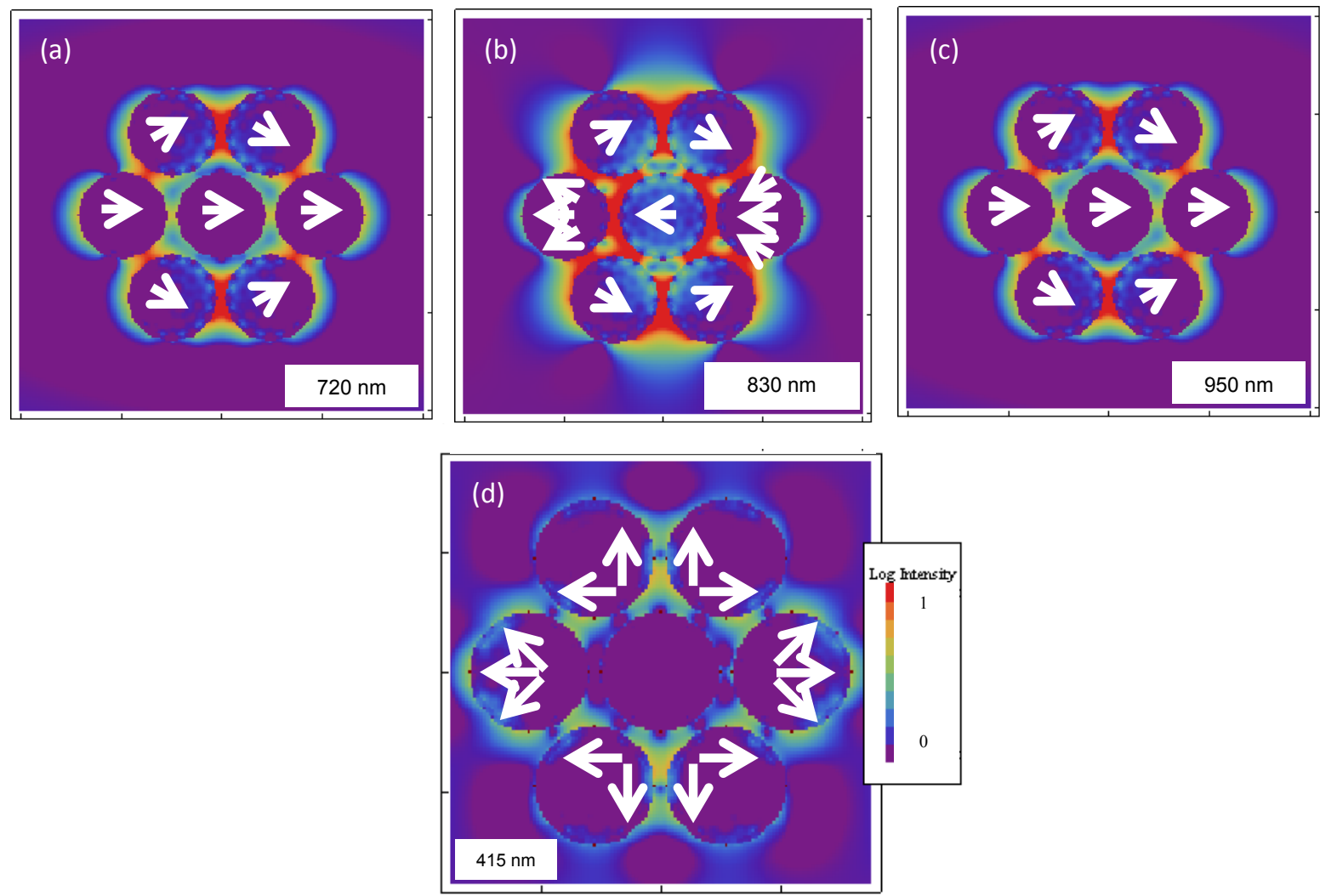

FIG. 3: Normalized log intensity plots showing the possible dipole distribution in the heptamers with a (a) radiant peak on one side of the Fano dip occurring at $720 \mathrm{~nm}$ (b) the subradiant dip at $830 \mathrm{~nm}$ clearly showing the central dipole oscillating out of phase in comparison to the ring monomers and (c) the radiant peak on the other side of the Fano dip occurring at $950 \mathrm{~nm}$ and (d) showing the multipolar peak that occurs at around $415 \mathrm{~nm}$ 
Symmetry breaking allows for greater SHG from standard plasmonic materials such as gold and silver, which are both centrosymmetric. Symmetric heptamers possess a $\mathrm{D}_{6 \mathrm{~h}}$ point group symmetry that can be easily modified in various ways. Here, we have looked at symmetry breaking using three techniques - the radius asymmetry, the center-displaced asymmetry and the changing the angle of incidence. Our simulations show that the Fano resonance gets significantly modified for our system in some cases, as further retardation effects come into play. For the symmetric standard heptamer though, it is expected from the symmetry that the spectral position of the dip and the two adjacent peaks is rather independent of the input polarization, and this was seen in the simulations (see Fig. 2). It can be seen from the figure $4 \mathrm{a}$, that the radius-asymmetry causes a slight blue-shift in the spectral position of the Fano dip while maintaining its scattering cross section; and it only reduces the scattering cross section of the peak without changing its spectral position significantly (Fig. 4b).
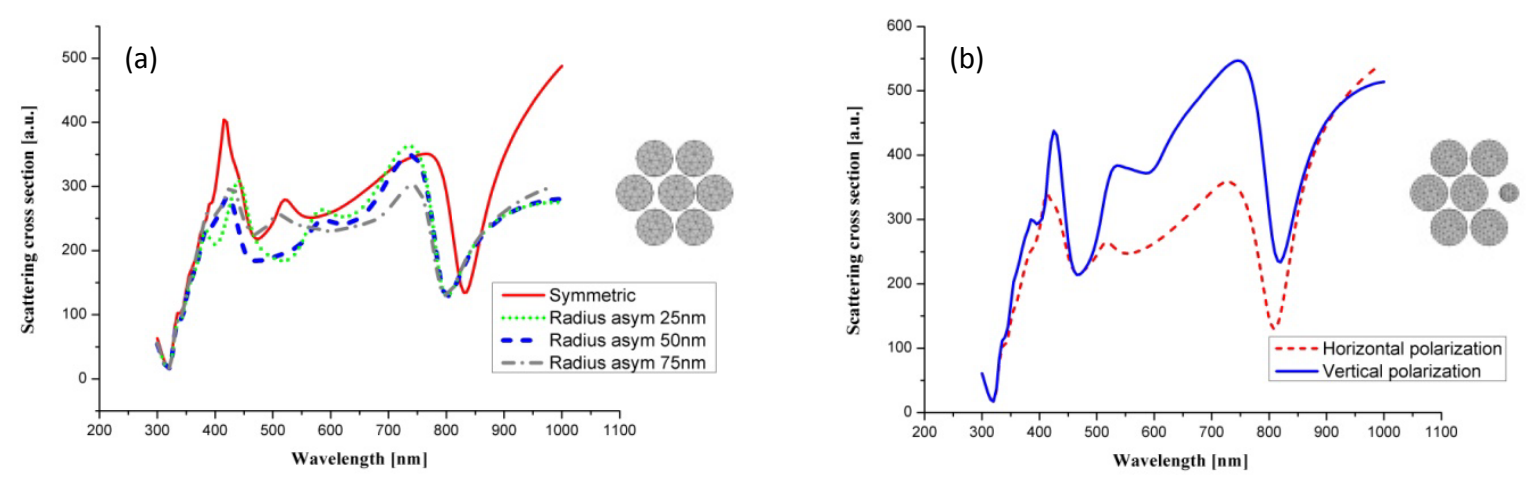

FIG. 4. Scattering cross sections of structures that suffer different asymmetries - (a) radius asymmetry and (b) center-displaced asymmetry of $10 \mathrm{~nm}$

Finer features are also modified. The inset shows the structure for a radius asymmetry of $50 \mathrm{~nm}$. A center-displaced asymmetry does not change the spectral position of the peak; however, for different polarizations, it shifts the entire curve up, making the bright mode more radiant, while making the dark mode less subradiant, which is not advantageous. By changing the angle of incidence from normal incidence (that has been used throughout this article) to various other angles, distorts the scattering cross section to a great extent, thereby preventing a radiant multipolar mode to be excited at the second harmonic. Therefore certain types of symmetry breaking are not preferred in this particular experimental setup as they do not improve the scattering cross section and thereby the second harmonic field radiated into the far field.

\section{FABRICATION AND EXPERIMENTS}

The heptamers were fabricated using electron beam lithography, followed by lift-off with PMMA as the photoresist. Examples of the fabricated structures are shown below in Fig. 5.

Some dark-field scattering measurements using an Olymp'us IX71 dark-field microscope and a spectrometer (Horiba Jobin Yvon, Triax 550) with liquid nitrogen cooled CCD were made on individual heptamers and structures which showed good fabrication parameter fit under the SEM, had the Fano dips where expected. An typical spectrum is shown in Fig. 6. Some preliminary tests were carried out with the fabricated heptamers using a standard setup for nonlinear measurements, consisting of a Ti:sapphire laser operating at a tunable wavelength range between $790 \mathrm{~nm}$ and $850 \mathrm{~nm}$, a photomultiplier tube linked to a lock-in detector for optimal signal to noise ratio and a spectrometer to look at the spectral emission range of the heptamers. Although the fabrication is yet to be completely optimized, the first tests hint that the heptamers show a significant SHG, as can be seen by the input power dependence of the output voltage measured by the PMT shown in Fig. 7. 

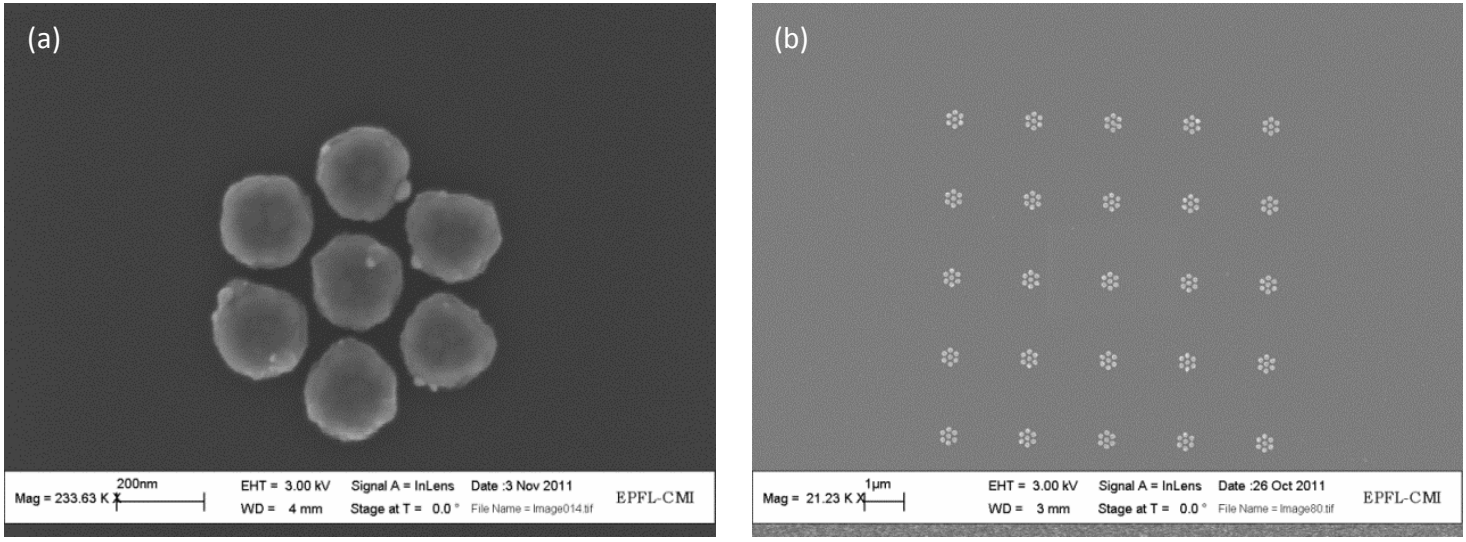

FIG. 5: A high resolution SEM (HRSEM) image of (a) a single heptamer unit and (b) a typical array of heptamers fabricated using electron beam lithography

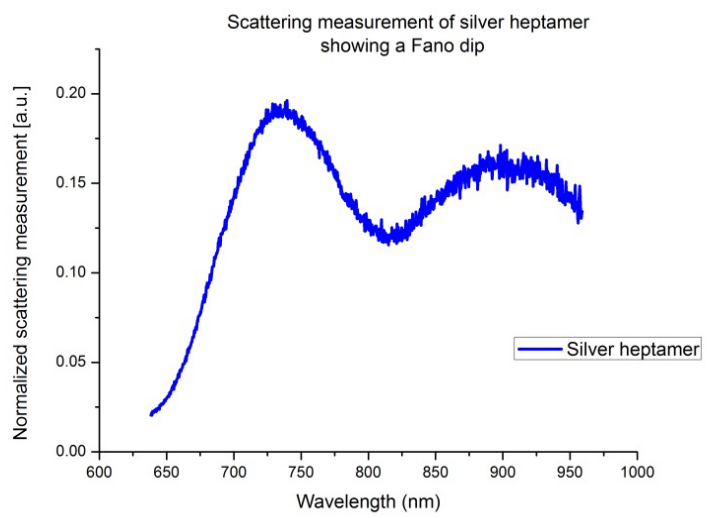

FIG. 6: Normalized scattering measurement of a fabricated silver heptamer with a diameter of $200 \mathrm{~nm}$ and a gap between two individual monomers as $30 \mathrm{~nm}$, showing a Fano dip at just about the required wavelength of the fundamental pump.

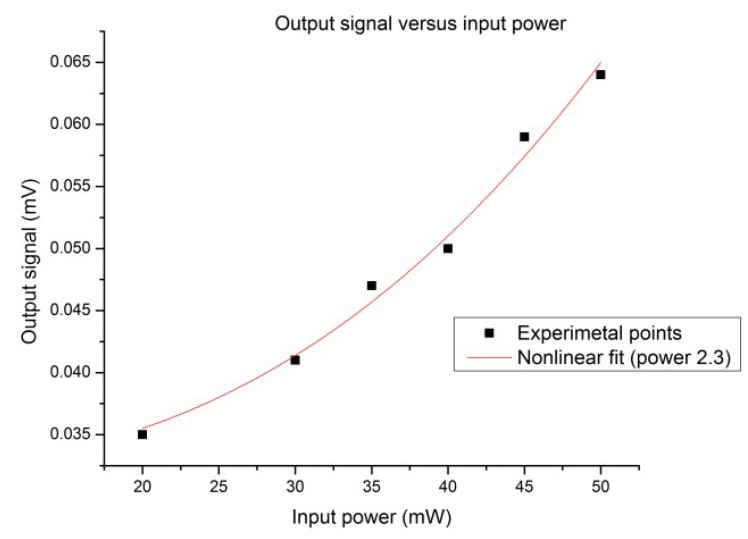

FIG. 7: Output signal from heptamers as measured with a PMT. The improper quadratic fit may be assigned to fabrication imperfections, which is being optimized. 


\section{CONCLUSIONS}

As a conclusion, we have shown in this work; that by an appropriate engineering of the plasmonic structures, one can take advantage of the fundamentally different nature of the a subradiant 'dark' mode and an adjacent radiant mode due to the excitation of higher order multipoles in silver heptamers to better control the conversion of the fundamental frequency into the second harmonic signal and its subsequent radiation into the far field. We are in the process of optimizing the fabrication of such structures and validating experimentally more concretely what has been proposed and studied numerically in this work. Further studies on such structures for more efficient SHG will be the endeavour of our future simulations and experimental work.

\section{ACKNOWLEDGMENTS}

Fruitful discussions with Dr. Christian Santschi are gratefully acknowledged. The work was supported by the Swiss National Science Foundation (project 200021_132694).

\section{REFERENCES}

[1] Fano, U., "Effects of configuration interaction on intensities and phase shifts", Phys. Rev., 124, 1866-1878 (1961)

[2] Miroshnichenko, A.E., Flach, S. and Kivshar, Y.S., "Fano resonances in nanoscale structures", Rev. Mod. Phys., 82, $2257(2010)$

[3] Fan, J.A., Bao, K., Wu, C., Bao, J., Bardhan, R., Halas, N.J., Manoharan, V.N., Shvets, G., Nordlander, P. and Capasso, F., "Fano-like interference in self-assembled plasmonic quadrumer clusters", Nano Lett., 10, 4680 (2010)

[4] Kern, A.M. and Martin, O.J.F., "Surface integral formulation for 3-D simulations of plasmonic and high permittivity nanostructures", J. Opt. Soc. Am. A, 26, 732 (2009)

[5] Hentschel, M., Saliba, M., Vogelgesang, R., Gissen, H., Alivisatos, A.P. and Liu, N., "Transition from isolated to collective modes in plasmonic oligomers", Nano Lett., 10, 2721 (2010)

[6] Hentschel, M., Dregely, D., Vogelgesang, R., Gissen, H. and Liu, N., "Plasmonic oligomers: the role of individual particles in collective behavior", ACS Nano, 5, 2042-2050 (2011)

[7] Lassiter, J.B., Sobhani, H., Fan, J.A., Kundu, J., Capasso, F., Nordlander, P. and Halas, N.J., "Fano resonances in plasmonic nanoclusters: geometrical and chemical tenability", Nano Lett., 10, 3184 (2010)

[8] Steele, J.M., Grady, N.K., Nordlander, P. and Halas, N.J., [Surface Plasmon Nanophotonics] Brongersma M.L. and Kik P.G. (eds.), Springer, 183 (2007)

[9] Prodan, E., Radloff, C., Halas, N.J. and Nordlander, P., "A hybridization model for the plasmon response of complex nanostructures", Science, 302, 419-422 (2003) 